\title{
Original articles \\ Effect of vitamin A adjunct therapy for cerebral malaria in children admitted to Mulago hospital: a randomized controlled trial
}

\author{
*Mwanga-Amumpaire J, Ndeezi G, Tumwine JK \\ Department of Paediatrics and Child Health, Makerere College of Health Sciences, P.O. 7072 Kampala, \\ Uganda
}

\begin{abstract}
Background: Malaria is a leading cause of mortality in Uganda accounting for $25 \%$ of deaths among children. Hitherto no adjunct therapy has been identified to improve outcome of cerebral malaria. Retinol suppresses growth of P. falciparum, scavenges free radicals, and exhibits synergistic action with quinine in parasite clearance.

Objective: To determine the effect of vitamin A supplementation on treatment outcome of cerebral malaria

Methods: In this randomised double-blind placebo controlled clinical trial we studied 142 children aged 6-59 months admitted with cerebral malaria in Mulago Hospital, Kampala. Children were randomised to either vitamin A or placebo and followed for 7 days. The main outcome measures were coma recovery time, time for convulsions to stop, and parasite and fever clearance. Secondary outcomes were overall mortality and time taken to start oral feeds.

Results: There was no difference in the coma recovery time $(\mathrm{p}=0.44)$, resolution of convulsions $(\mathrm{p}=0.37)$, fever clearance $(\mathrm{p}=0.92)$, parasite clearance $(\mathrm{p}=0.12)$, and starting oral feeds between the two treatment groups. Mortality was higher $(16.2 \%)$ in the placebo than in the vitamin A group (8.1\%): RR 1.4; 95\% CI 1.0-2.1.

Conclusions: Vitamin A as adjunct therapy did not significantly reduce coma duration but there were fewer deaths in the vitamin A arm.

African Health Sciences 2012; 12(2): 90 - 97 http://dx.doi.org/10.4314/ahs.v12i2.3
\end{abstract}

\section{Introduction}

Malaria is responsible for up to 2.7 million deaths every year and remains the major cause of morbidity and mortality worldwide. ${ }^{1,2}$ Malaria is the leading cause of morbidity and mortality in Uganda and is endemic in $95 \%$ of the country, with the exception of mountainous areas in the southwest, west, and eastern parts. Overall, malaria accounts for $30-50 \%$ of all the outpatient visits at health facilities, $30 \%$ of hospital admissions as well as $25-30 \%$ of deaths among children less than five years of age in highly

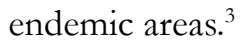

Recent studies have shown improved outcome with artesunate compared to quinine in treatment of severe malaria. However, mortality from cerebral malaria remains high. 4,5 Alternative ways for treatment aiming at reversing or ameliorating processes that may contribute directly or indirectly to a fatal outcome are needed. Several adjunctive

*Correspondence author:
Juliet Mwanga-Amumpaire
Department of Paediatrics and Child Health
Mbarara University of Science and Technology
P.O. Box 1410
Mbarara, Uganda
E-mail: mwangajuliet@gmail.com

90 therapies have been tried, targeted at reversing the pathophysiological processes that lead to cerebral malaria. However no single adjunct therapy has yet shown clear efficacy in improving the outcome of treatment of cerebral malaria. ${ }^{6}$

There is hope that vitamin A may be of use as in vitro studies have shown that retinol levels corresponding to normal serum levels can suppress growth of Plasmodium falciparum ${ }^{7}$ while community based studies have shown significant reduction in malaria episodes in children in whom vitamin A was supplemented. ${ }^{8}$ The real immunogenic mechanisms of action of vitamin $\mathrm{A}$ is not yet clear. It enhances immunity against malaria, reduces secretion of Plasmodium falciparum-induced Tumour Necrosis Factor (TNF) by monocytes and macrophages, acts synergistically with some antimalarials such as quinine to reduce parasite replication, ${ }^{9,10,11}$ and has shown antioxidant activity in other tissues. ${ }^{12}$

Cerebral malaria is thought to result from increased concentrations of proinflammatory cytokines induced by $P$. falciparum such as TNF, and the exaggerated effects of free oxygen radicals produced by the phagocytes on host tissues. Unfortunately vitamin A deficiency among children 
is widespread in the malaria- endemic areas of Africa. In Uganda, severe clinical vitamin A deficiency ranges from $4-6.6 \%$ among children aged $0-5$ years while subclinical deficiency approaches fifty four percent. ${ }^{13}$ Acute falls in serum retinol levels to 1.0ìmol/L have been described in acute malaria attacks. ${ }^{14}$

Though community based studies have shown the benefit of supplementing vitamin $A$ in reducing morbidity from malaria, ${ }^{8}$ only one clinical trial on Mozambican children has been described in severe malaria in which a reduction in risk of death was noted. ${ }^{15}$ However, the whole spectrum of severe malaria and not just cerebral malaria was considered in the Mozambican study. We report results of a randomized controlled trial of vitamin $\mathrm{A}$ as adjunct therapy for childhood cerebral malaria in Uganda.

\section{Methods}

This was a double blind placebo-controlled randomized clinical trial carried out from May 2002 to February 2003 in Mulago Hospital, the national referral and teaching hospital. Patients were enrolled from the Paediatric Emergency Unit (Acute Care Unit) and followed up on one of the four general paediatric wards until discharge. The study population comprised children aged 6-59 months admitted with a confirmed diagnosis of cerebral malaria whose caretaker gave informed consent to participate in the study. Cerebral malaria was defined as unarousable coma lasting more than 30 minutes following cessation of a seizure with peripheral asexual P.falciparum parastemia and absence of other causes of coma.

We excluded children who had received vitamin A within the last 3 months and those whose conditions warranted treatment with vitamin $A$ such as persistent diarrhoea, severe pneumonia, severe malnutrition, and overt clinical vitamin A deficiency.

\section{Randomization}

Randomization was done in blocks of variable sizes (4-10) using a set of computer generated random numbers. All study participants received intravenous quinine for treatment of severe malaria and were randomized to receive either vitamin A or placebo as adjunct therapy. Each block contained equal numbers of patients receiving vitamin $\mathrm{A}$ and those receiving the placebo.

\section{Blinding}

The randomisation sequence was generated by an independent statistician not involved in the study design, data processing or analysis. Instructions for treatment were produced according to the randomization scheme and placed in opaque sealed envelopes with unique identification numbers by an independent pharmacist not involved in the study. Sealed codes were kept safely by an independent doctor in the paediatrics department and were opened at the completion of data analysis. The envelopes were kept in a locked cabinet in the Acute Care Unit (ACU). Following enrolment of an eligible patient, the researcher/research assistant then picked the next available envelope containing the treatment protocols and handed it to the treatment nurse, who then administered the drugs to the patient. The investigators, nurses and caretakers were blinded to group assignments.

\section{Allocation concealment}

The randomization code was sent to the manufacturer (RpScherer, S.P.A., Aprilia, LTD) for labelling of the drug bottles. The vitamin A capsules and placebo were exactly the same in colour, shape and size. Treatment codes were placed in opaque sealed envelopes and these were only opened after an eligible participant was enrolled.

\section{Sample size and statistical issues}

We calculated the sample size of 68 patients in each group for $80 \%$ power and $95 \%$ confidence intervals, with an assumption that $41 \%$ of the children would have regained consciousness within the first 24 hours and this would increase to $66.4 \%$ with vitamin $\mathrm{A} .{ }^{16}$

\section{Data management and analysis}

Data entry and analysis were done using SPSS 11.0 statistical software package. Analysis was by 'intention to treat'. Data were summarised in form of means with standard deviations and percentages. The Chisquared test and Fisher's exact test with corresponding odds and risk ratios where appropriate, and 95\% confidence intervals were used to compare categorical data. Associations whose pvalues were $<0.05$ were considered significant. Interaction and joint effects of risk factors on outcome were assessed using logistic regression. Kaplan-Meier survival analysis and the Log rank test were used to assess the effect of vitamin A on survival.

\section{Measurements}

The main outcome measures were coma recovery time as determined by a Blantyre coma score of 5 time taken for convulsions to stop after initiating treatment and time to sit unsupported. Other 
outcomes included, fever clearance time (that is time taken for the temperature to fall below $37.5^{\circ} \mathrm{C}$ and remain so for at least 24 hours), parasite clearance time (that is time for the peripheral blood slide to have 0 parasites/il of blood), time taken to start oral feeds, and death.

\section{Treatment}

All patients received intravenous quinine dihydrochloride $10 \mathrm{mg}$ salt $/ \mathrm{kg}$ body weight in 10 $\mathrm{mls} / \mathrm{kg}$ of $5 \%$ dextrose, which was infused over 24 hours every 8 hours until the patient regained consciousness or was able to take oral drugs. Quinine sulphate was then given orally $(10 \mathrm{mg}$ salt $/ \mathrm{kg}$ ) every 8 hours until seven days of treatment were completed.

Children assigned vitamin A received a single dose of 100,000 International Units (I.U) for those below 1 year and 200,000I.U for those above one year of age. The vitamin A capsules contained 200,000 International Units of vitamin A (as retinyl palmitate) and 40 International units of vitamin $E$ to stabilise the vitamin A and increase its shelf life, in soybean oil as excipient. The placebo contained 40 International units of vitamin $\mathrm{E}$ (as dl-tocopherol) in soybean oil as excipient. The vitamin A capsules and placebo were donated by the Task Force Sight and Life based in Basel, Switzerland and were manufactured and packaged by RpScherer, S.P.A., Aprilia (LT), Italy. Administration was via a nasal gastric tube by the treatment nurse. The vitamin A was drawn from the capsule using a small needle and pushed down the nasal gastric tube and the tube was rinsed with clean warm water. All patients received $25 \%$ dextrose $(2 \mathrm{ml} / \mathrm{kg})$ as a bolus for resuscitation and to prevent hypoglycaemia. Milk with sugar was administered via a nasal gastric tube at a 2 hourly interval until the child was able to take oral feeds. Signs and symptoms of vitamin A toxicity such as excessive vomiting, bulging fontanelle in children below 18 months, headache in older children and skin rash were sought for.

\section{Ethical considerations}

Permission to carry out the study was obtained from the Department of Paediatrics and Child Health, the Makerere University College of Health Sciences Research and Ethics Committee and the Uganda National Council for Science, and Technology. Written informed consent was given by the parents or guardians.

\section{Results}

Four hundred and thirty six children with cerebral malaria were seen in the ACU of Mulago hospital. Of these, 142 were recruited into the study. Among those recruited 74 were randomly assigned to receive vitamin A while 68 received the placebo (figure1).

\section{Figure 1: The trial profile}

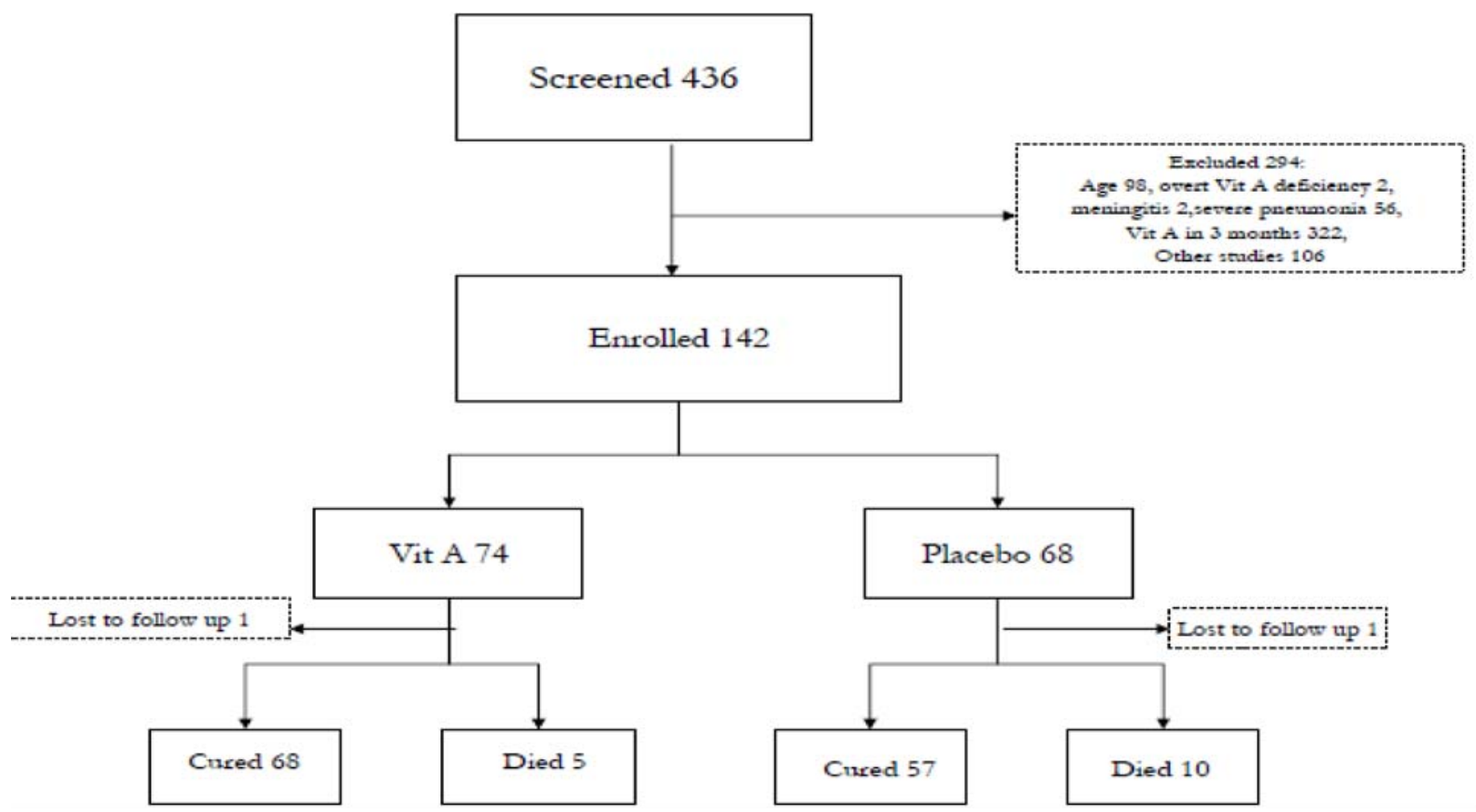


Of the 142 children, $65(45.8 \%)$ were females. Among the females, 34 (52.3\%) received vitamin A while $31(47.7 \%)$ received placebo. Forty $(51.95 \%)$ of the males received vitamin A and 37 (48.05\%) received the placebo. Baseline characteristics of patients in the two study arms were comparable as shown in table 1.

\section{Baseline laboratory findings}

Children in the placebo group had higher mean parasite densities than those who received vitamin A. However only $12(8.5 \%)$ of the children had parasite counts $\geq 500,000 / \mathrm{ul}$.
Four of these were in the vitamin A group while 8 were in the placebo group. Baseline laboratory characteristics are shown in table 2.

\section{Clinical response}

There were no statistically significant differences in the outcome measures between the two treatment arms as shown in table 3. There was no significant difference in survival or occurrence of neurological sequelae in the two treatment groups.

Table 1: Baseline clinical characteristics of 142 children admitted with cerebral malaria

\begin{tabular}{|c|c|c|c|}
\hline Variable & $\begin{array}{l}\text { Vitamin } \mathbf{A}=74 \\
\text { No of children }(\%)\end{array}$ & $\begin{array}{l}\text { Placebo }=68 \\
\text { No of children }(\%)\end{array}$ & $\mathrm{p}$-value \\
\hline Male sex & $40(51.9)$ & $37(48.1)$ & 1.000 \\
\hline Blantyre Coma Score 1 & $22(29.7)$ & $17(25.0)$ & 0.528 \\
\hline Blantyre Coma Score 2 & $52(70.3)$ & $51(75.0)$ & 0.528 \\
\hline Convulsions & $71(95.9)$ & $65(95.6)$ & 1.000 \\
\hline \multicolumn{4}{|l|}{ Frequency of seizures } \\
\hline$<5 / 24 \mathrm{hrs}$ & $35(49.3)$ & $37(56.9)$ & 0.373 \\
\hline$\geq 5 / 24 \mathrm{hrs}$ & $36(50.7)$ & $28(43.1)$ & \\
\hline Fever & $73(98.6)$ & $68(100)$ & 1.000 \\
\hline Vomiting & $50(67.6)$ & $44(66.2)$ & 0.719 \\
\hline Difficult breathing & $8(10.8)$ & $8(11.8)$ & 1.000 \\
\hline Antimalarial drugs & $60(81.1)$ & $50(73.5)$ & 0.282 \\
\hline Abnormal posture & $31(41.9)$ & $39(57.4)$ & 0.066 \\
\hline Abnormal tone & $64(86.5)$ & $61(89.7)$ & 0.300 \\
\hline Hepatomegally & $65(87.8)$ & $53(77.9)$ & 0.116 \\
\hline Splenomegally & $51(68.9)$ & $38(55.9)$ & 0.109 \\
\hline${ }^{\mathrm{T}}$ Severe anaemia & $16(21.6)$ & $18(26.5)$ & 0.499 \\
\hline Parasite density & $4(5.4)$ & $8(11.8)$ & 0.231 \\
\hline
\end{tabular}

Table 2: Baseline laboratory indices in children admitted with cerebral malaria, May 2002 to February 2003

\begin{tabular}{lllccc}
\hline Variable & $\begin{array}{l}\text { Vitamin A, N=74 } \\
\text { Mean (SD) }\end{array}$ & Range & $\begin{array}{l}\text { Placebo, N- 68 } \\
\text { Mean (SD) }\end{array}$ & Range & p-value \\
\hline HB & $6.3(2.1)$ & $1.8-10.5$ & $6.0(2.1)$ & $2.1-11.1$ & 0.36 \\
WBC x 10 & $10.7(8.9)$ & $1.7-58.5$ & $8.2(4.4)$ & $1.5-26.5$ & $0.036^{*}$ \\
Lymphocytes (\%) & $37.5(14.3)$ & $10.0-78.0$ & $38.8(16.4)$ & $10.0-92.0$ & 0.61 \\
Neutrophils (\%) & $61.4(14.5)$ & $20.0-90.0$ & $59.4(16.5)$ & $8.0-88.0$ & 0.45 \\
Platelets x 10 & $158.6(98.6)$ & $308-522$ & $173.8(102.3)$ & $15.0-668.0$ & 0.37 \\
Parasite density x & $116.8(210.1)$ & $0.641-267$ & $254.5(450.8)$ & $0.064-2.43$ & $0.019^{*}$ \\
\hline
\end{tabular}

$10^{3} / \mu 1$

*p-values less than 0.05 considered significant 
Table 3: Outcome measures of children supplemented with vitamin A or placebo as adjunct treatment for cerebral malaria

\begin{tabular}{llll}
\hline Outcome & $\begin{array}{l}\text { Vitamin A, } \\
\text { N=68 } \\
\text { Mean time (SD) } \\
\text { in hrs }\end{array}$ & $\begin{array}{l}\text { Placebo, } \\
\mathbf{N = 5 7} \\
\text { Mean time (SD) } \\
\text { in hrs }\end{array}$ & p-value \\
\hline Coma resolution time & $46.9(28.5)$ & $43.5(19.1)$ & 0.44 \\
Resolution of convulsions & $32.86(13.7)$ & $35.35(16.6)$ & 0.37 \\
Fever clearance time & $52.24(26.8)$ & $51.79(20.2)$ & 0.92 \\
Parasite clearance time & $57.53(22.7)$ & $63.65(20.6)$ & 0.12 \\
Feeding orally & $57.94(33.3)$ & $50.11(20.9)$ & 0.13 \\
\hline
\end{tabular}

${ }_{\mathrm{p}}^{\mathrm{p}}<0.05$ are considered significant

Figure 2: Kaplan Meier curve for mortality by treatment group among 142 children admitted with cerebral malaria

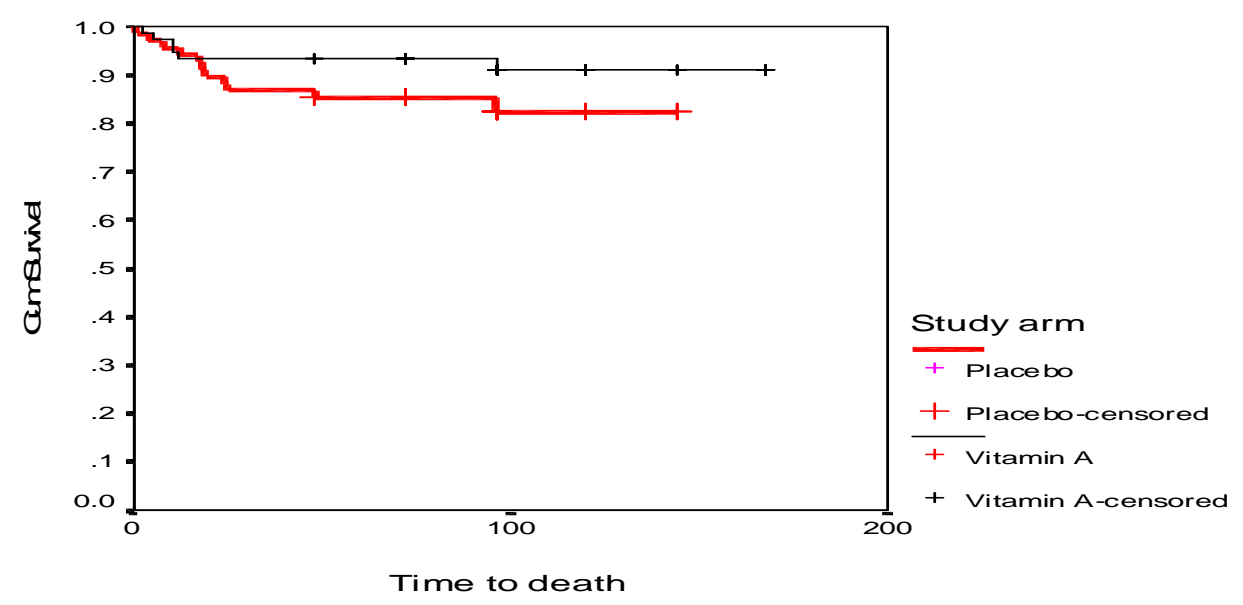

\section{Mortality}

Overall 15 children died, and 2 were lost to follow up. Since the analysis was by intention to treat the 2 lost to follow up were also assigned the worst outcome, which in this case is death. This brought the total number of the children that died to 17 and an overall mortality of $12 \%$. Children who did not receive vitamin A were twice as likely to die $(16.2 \%)$ compared to those who received vitamin $\mathrm{A}(8.1 \%)$. However the difference was not statistically significant (Log rank test, $\mathrm{p}=0.135)$.

\section{Regression analysis}

Linear regression analysis did not reveal any factor identified to influence outcome measures, such as time to regain consciousness, parasite clearance time, fever clearance time, and resolution of convulsions. Using the backward Wald model of logistic regression analysis, no variables were found to affect mortality among children who received vitamin A.

\section{Adverse drug events}

No gastroenterological, dermatological, or neurological adverse drug events were detected. In particular, clinical signs of raised intracranial pressure, excessive vomiting and skin rash were not seen among the study subjects.

\section{Discussion}

This study aimed at determining the effect of vitamin A supplementation on treatment outcome of cerebral malaria.

\section{Time to regain consciousness}

In this study, there was no difference in the coma recovery time between the two treatment groups. Our results are similar to findings of a study carried out in the Central Hospital of Maputo, Mozambique, where vitamin A adjunct therapy did not influence coma recovery time. ${ }^{15}$ The mechanisms through which coma occurs in cerebral malaria are not 
known, but, it is believed that many factors interact to cause this coma. These factors include sequestration of parasitised red blood cells within cerebral venules and depression of the reticular activating system within the brainstem and cerebrum. It is also thought that toxins produced by the sequestered parasites stimulate host cells to produce substances that interfere with neuronal metabolism and cause abnormalities of neurotransmitter synthesis, release and binding. ${ }^{17,18}$ It is possible that Vitamin A which acts by blocking production of pro-inflammatory cytokines $^{11,19}$ and is a scavenger of free radicals, 20 does not counteract all the factors that have been implicated in causation of coma.

It is also possible that the dose of vitamin A used in our study was too small to have an effect since studies in other disease conditions showed that higher doses $(400,000 \mathrm{IU})$ had more impact than lower doses. ${ }^{21}$ In this study, because of fear overdose we could not give larger doses of vitamin A since the serum retinol levels were not monitored. Since in an acute malaria attack vitamin A together with its transporting proteins, retinol binding protein and prealbumin are reduced secondary to the increase in interleukin-6, some authors have suggested that in order for vitamin A to have an effect it should be administered with a transporter. ${ }^{19}$

\section{Resolution of convulsions}

The time taken for convulsions to stop was shorter in the vitamin A group than in the placebo group. Recurrent convulsions are a poor prognostic indicator in cerebral malaria ${ }^{16,17,22-24}$. Therefore, this difference of 2.43 hours for convulsions to stop between children that received vitamin $\mathrm{A}$ and those who received placebo may be of clinical importance. The earlier seizures stop the better because this will minimise complications that arise due to repeated seizures for example intracranial hypertension, hypoxia, neuronal damage and aspiration of gastric contents and depression of the respiratory center. The exact mechanisms through which seizures occur are not known and therefore the mechanisms through which vitamin A acts to reduce seizures are not known.

It is believed that TNF plays a role in pathogenesis of seizures because its levels are high in cerebral malaria. ${ }^{17,25}$ The TNF triggers a cytokine cascade that leads to production of free radicals, which cause insult and inflammation in a critical part of the brain..$^{25}$ Perhaps vitamin A acts through its antioxidant properties and by blocking synthesis of TNF. ${ }^{10}$

\section{Fever clearance}

In this study there was no difference in fever clearance time between the two treatment arms. This is comparable to other studies in children and adults ${ }^{14}$, ${ }^{15}$ A community study in Papua New Guinea showed less fever episodes in the vitamin A supplemented group compared to the placebo group probably because the mechanism of action in this study was through preventing malaria infection rather than fever clearance. ${ }^{8}$

\section{Parasite clearance}

Parasite clearance was earlier in the vitamin A group by 6 hours compared to the placebo group, $(p=0.120)$. These results are comparable to other studies; a study done on Vietnamese adults ${ }^{14}$ showed a trend to faster clearance of parasites among those who received vitamin A. A community based study done in Papua New Guinea ${ }^{8}$ found significantly lower positive blood slides in the vitamin A group compared to the placebo group. Several mechanisms have been put forward through which vitamin A may act in parasite clearance. Vitamin A is known to act synergistically with quinine in killing malaria parasites, inhibits growth and replication of Plasmodium falciparum in vitro, and promotes nonopsonic phagocytosis of parasitised red blood cells. ${ }^{7,9,10,11,}$

\section{Mortality}

The overall mortality of $12 \%$ is comparable to $7-17$ $\%$ reported in other studies done in Mulago in Uganda, ${ }^{16,26,}$ other African countries ${ }^{23,27-29}$ and in Papua New Guinea. ${ }^{30}$ Nevertheless, in our study, deaths in the vitamin A group were less than those in the placebo group although this study did not have sufficient power to examine the effect of vitamin A on mortality. This figure may be of clinical significance and is consistent with results in a Mozambican study ${ }^{15}$ where vitamin A was found to reduce deaths significantly after the first 5 hours $(p=0.015)$. There is evidence that vitamin A supplementation reduces mortality and morbidity among children. ${ }^{21,31,32}$ Although the actual mechanism by which vitamin A reduces malaria deaths is not known, vitamin A reduces production of antiinflammatory cytokines such as TNF and has antiplasmodial action. ${ }^{9,10,19}$

\section{Adverse drug events}

No adverse drug events were detected in this study. This possibly means that the dose of vitamin A used 
in our study is safe. Whether this dose is enough to show pharmacological efficacy needs further investigation.

\section{Study limitations}

It was not possible to determine serum retinol levels at baseline and the consequent levels during treatment so as to relate them to the outcome. Investigations to exclude certain important prognostic indicators such as $\mathrm{pH}$, blood cultures, and serum lactate were not done. Since the sample size was small mortality could not be taken as a primary outcome.

\section{Conclusion}

We therefore conclude that vitamin $\mathrm{A}$ as adjunct therapy did not significantly reduce coma duration. There was a trend to earlier resolution of convulsions and less mortality in children with cerebral malaria in the vitamin A supplemented group. We recommend a larger study with sufficient power to study the effect of vitamin A on outcome of cerebral malaria especially mortality and a safety/dose finding study comparing the dose used in this trial with higher doses.

\section{Acknowledgements}

We are grateful to the following organizations for the financial support: Ministry of Education through the Uganda Independence Scholarship, Mbarara University, Uganda Malaria Surveillance Project through Makerere University-University of California Sanfrancisco collaboration (MU-UCSF), Regional Center for Quality of Health Care (RCQHC) and Nuffield foundation. Task force Sight and Life based in Basel Switzerland donated the vitamin $A$ and the placebos that were used in this study. We also thank all the nursing staff of Jelliffe ward and laboratory technicians that participated in the study.

\section{References}

1. World Health Organisation Malaria Report (2008) Geneva

2. WHO. Severe and complicated malaria. Trans R Soc Trop Med Hyg 1990; 84 (suppl 2): 1-65.

3. Uganda National Malaria Control Policy, Ministry of Health, Kampala, Uganda 20052010.

4. Dondorp AM, Fanello CI, Hendriksen IC, Gomes E, Seni A, Chhaganlal KD, et al. Artesunate versus quinine in the treatment of severe falciparum malaria in African children
(AQUAMAT): an open-label, randomised trial. Lancet. 2010 Nov 13;376(9753):1647-57.

5. Dondorp A, Nosten F, Stepniewska K, Day N, White NJ, for the South East Asian Quinine Artesunate Malaria Trial (SEAQUAMAT) group. Artesunate versus quinine for treatment of severe falciparum malaria: a randomised trial. Lancet 2005; 366: 717-25.

6. Adjunctive therapy for cerebral malaria and other severe forms of Plasmodium falciparum malaria Chandy C John1, Elizabeth Kutamba, Keith Mugarura, and Robert O Opoka. Expert Rev Anti Infect Ther. 2010 September ; 8(9): 997 1008.

7. Davis T M; Skinner A T S, Beilby J. In vitro growth inhibition of Plasmodium falciparum by retinol at concentrations present in normal human serum. Acta Trop 1998; 69:111-9.

8. Shankar A H, Genton B. Effect of Vitamin A supplementation on morbidity due to plasmodium falciparum in young children in Papua New Guinea: a randomised trial. Lancet 1999; $354:$ 203-9.

9. Serghides L, Kain K C. Peroxisome Proliferator - Activated Receptor Ò-Retinoid X Receptor Agonists increase CD 36 - Dependant Phagocytosis of plasmodium falciparum parasitized erythrocytes and decrease malaria induced TNF - á Secretion by Monocytes/ Macrophages. J. Immunol 2001; $166: 6742-8$.

10. Serghides L, Kain K.C. Mechanism of protection induced by vitamin A in falciparum malaria. Lancet 2002; 359:1404-06

11. Old 10 Skinner A T, Barrett H. Heterogenous activity in vitro of Vitamin A (retinol) in combination with novel established antimalarial drugs. Trans R Soc Trop Med Hyg. 1999; 93 (5) 550-1.

12. Susan A. Keys and William F. Zimmerman. Antioxidant Activity of Retinol, Glutathione, and Taurine inBovine Photoreceptor Cell MembranesExp. Eye Res. (1999) 68, 693-702

13. Global prevalence of vitamin A deficiency. Micronutrient deficiency information system. WHO. MIDS working paper \# 2; 1995.

14. Davis T M, Binh TQ, Thu LT, Rossi R, Danh PT, Barrett PH et al. Pharmacokinetics of Retinyl palmitate and retinol after intramuscular retinyl palmitate administration in severe malaria. Clin Sci 2000; 99:433-441.

15. Varandas L, Julien M, Gomes A, Rodrigues P, Van Lerberghe W, Malveiro F, et al. A 
randomised, double blind, placebo controlled clinical trial of Vitamin A in severe malaria in hospitalised Mozambican children. Ann Trop Paed 2002;2201: 211-222.

16. Idro R, Karamagi C, Tumwine J. Immediate outcome and prognostic factors for cerebral malaria among children admitted to Mulago Hospital, Uganda Ann Trop Paediatr. 2004 Mar;24(1):17-24.

17. Newton C R, Krishna S. Severe falciparum malaria in children: Current understanding of pathophysiology and supportive treatment. Pharmacol-Therapeut. 1998; 79: 1-53.

18. WHO (2000) Severe falciparum malaria. Trans Roy Soc Trop Medicine and Hyg, 94, supplement 1.

19. Tabone M D, Mwanza K, Ja-del C. The role of interleukin-6 in Vitamin A deficiency during plasmodium falciparum malaria and possible consequences for Vitamin A supplementation. Immunology 1992; 75: 553-4.

20. Keusch G T. Micronutrients and susceptibility to infection. Ann NY Acad Sci. 1990; 587:1818.

21. Hussey G D, Klein M. A randomised controlled trial of vitamin $A$ in children with severe measles. New Eng J Med 1990;323:160-4

22. Idro R, Jenkins NE, Newton CR. Pathogenesis, clinical features, and neurological outcome of cerebral malaria. Lancet Neurol 2005; 4: 827-40

23. Molyneux M E, Taylor T E, Wirima J J, Harper G. Clinical features and prognostic indicators in peadiatric cerebral malaria: a study of 131 comatose Malawian children. Quart J Med 1989;71: 441-459.

24. Jaffer S, Van Hensbroek N B, Palmer A, Schneder G, Greenweel B. Predictors of fatal outcome following childhood cerebral malaria. Amer $J$ Trop Med Hyg 1997; 57: 20-4.
25. Kwiatkowski D, Hill AV, Sambou I, Twumasi P, Castracane J, Manogue KR,et al. TNF concentration in fatal cerebral malaria, non-fatal cerebral malaria and uncomplicated Plasmodium falciparum malaria. Lancet 1990; 336:1201-04.

26. Aceng JR, Byarugaba JS, Tumwine JK. Rectal artemether versus intravenous quinine for the treatment of cerebral malaria in children in Uganda: randomised clinical trial. BMJ. 2005;330:334. doi: 10.1136/bmj.330.7487.334.

27. Brewster D R, Kwiatkowski D, White N J. Neurological sequalae of cerebral malaria in children. Lancet, 1990;336: 1039-1043.

28. Marsh K, Forster D, Waruiru C, Mwangi I, Winstanley M, Marsh V, et al. Indicators of life threatening malaria in African children. New Eng J Med 1995; 332: 1399-404.

29. Carme B, Bouquety J C, Plassart H. Mortality and sequelae due to cerebral malaria in African children in Brazzaville, Congo. Amer J Trop Med Hyg 1993; 48:216-21.

30. Genton B, al-Yaman F, Alpers M P, Mokela D. Indicators of fatal outcome in paeditric cerebral malaria: A study of 134 comatose Papua New Guinean children. Internat J Epidemiol 1997;26:670-6

31. Mayo-Wilson E, Imdad A, Herzer K, Yakoob MY, Bhutta ZA. Vitamin A supplements for preventing mortality, illness, and blindness in children aged under 5: systematic review and meta-analysis. BMJ. 2011 Aug 25;343:d5094

32. Imdad A, Yakoob MY, Sudfeld C, Haider BA, Black RE, Bhutta ZAImpact of vitamin A supplementation on infant and childhood mortality. BMC Public Health. 2011 Apr 13;11 Suppl 3:S20. 